\title{
Lernen wir aus der Geschichte?
}

Online publiziert: 18. Januar 2013

(c) Die Autor(en) 2013. Dieser Artikel ist auf Springerlink.com mit Open Access verfügbar.

\section{Liebe Kolleginnen und Kollegen!}

In diesem Heft finden Sie einen hervorragenden Überblick über die Geschichte der Universitäts-Augenklinikin Wien über die ersten 150 Jahre. Frau Dozentin SchmidtWyklicky und Herr Dr. Helmut Gröger entfalten präzise die Entwicklung der Wiener Ophthalmologie aus historischer Sicht.

Aus klinischer Sicht macht es mich stolz, 23 Jahre (1972-1995) an dieser Augenklinik tätig gewesen zu sein, zuletzt auch als stellvertretende Leiterin der 1. Univ.Augenklinik (Abt. B) unter der Leitung von Professor Dr. Heinz Freyler. Über die Aktivitäten der von den beiden Historikern nicht abgedeckten letzten 50 Jahre der Augenklinik Wien zwischen 1955 bis 2005 werden Sie einen Überblick im nächsten Heft 1/2013 des spektrum der augenheilkunde nachlesen können.

Verlässt man aber die Aufreihung großer Entdeckungen, Innovationen, Internationalität und Entwicklungen neuer chirurgischer Techniken, dann wiederholen sich im Schatten von Wissenschaft, Lehre und Praxis auch immer wieder Intrigen, Neid und Bosheit als sogenannter „wissenschaftlicher bypack“.

So hat - nur um ein Beispiel zu nennen - Barth über viele Jahre Beer nur als Zeichner beschäftigt und tat alles, um Beer in seiner Entwicklung in der Ophthalmologie zu hindern. Operieren musste sich Beer mühsam selbst beibringen, obwohl Barth selbst ein bereits anerkannter Kataraktchirurg war. Beer benötigte 25 Jahre, um sein Ziel - die Anerkennung der Ophthalmologie als eigenes Fach - zu verwirklichen. Genau genommen dauerte es noch einmal 6 Jahre, als am 2. Mai 1818 endlich die Ophthalmologie zum ordentlichen Lehrfach und die 1. Univ.Augenklinik zum Ordinariat erhoben wurde. Ein Jahr später war Beer bereits so schwer krank, dass er seine Tätigkeit nicht mehr ausführen konnte. Im Hintergrund all dieser Verzögerungen wirkte effizient ein Mann der Universität, erster Leibarzt und Promedicus A. J. Freiherr von Stifft, der seit 1803 auch als Vizerektor tätig gewesen war und sich Neuerungen nur ungern aufschloss. So förderte er auch Rosas als Nachfolger Beers, der dafür eigentlich seinen Schwiegersohn, den begabten Friedrich Jäger von Jaxtthal vorgesehen hatte.

Rosas erfüllte seine Klinikleitung mit viel Konservatismus über 33 Jahre und war seinem Gönner Stifft so sehr ergeben, dass er sich der ,jungen Wiener Schule“ um Rokitanski und Skoda nicht anschließen konnte, was auch in der Ablehnung einer Stellenverlängerung von Ignaz Semmelweis gipfelte.

Glücklicherweise war Ferdinand Ritter von Arlt ein Mann der Innovation und Internationalität. Hatte Beer 1813 bereits über die „Brillenwuth berichtet“, also das Tragen einer Brille nur aus modischen Gründen, ein Trend, der im letzten Jahrzehnt als „Harry Potter Syndrom" bezeichnet wurde, so widmete sich Arlt der Pflege gesunder und kranker Augen und stellte in seiner 1846 veröffentlichen Aufklärungsschrift erstmals die Forderung auf, dass die Brillenbestimmung nur durch Augenärzte vorgenommen werde sollte. Ein Wunsch, über den sich heute jeder Präsident oder Präsidentin der Österreichischen Ophthalmologischen Gesellschaft freuen würde, auch wenn wir wissen, dass dieser Kampf schon lange verloren ist.

Gemeinsam mit seinem begabten Schüler Albrecht von Graefe und Frans Cornelius Donders gründete Arlt 1884 das „Archiv für Ophthalmologie“, das heute als „Graefes Archives for Clinical and Experimental Ophthalmology“ jedem bekannt ist.

Parallel zu all seinen Innovationen war diese Zeit aber vom Kampf zwischen Arlt Schülern und Jäger Schülern (dem Enkel Beers) geprägt. Arlt wurden durch einen Plan des Staatssekretärs im Ministerium für Cultus und Unterricht, Graf Thun Hohenstein, nur 20 der 50 Betten der Augenklink übergeben, da die Gründung der 2. Univ.Augenklinik anstand, die mit Eduard Jäger besetzt werden sollte. Ein Unterfangen, dem sich Arlt bis zu seiner Emeritierung zähe widersetzte.

Erst als Stellwag Arlt nachfolgte, wurde 1883 die 2. Univ.-Augenklinik mit Jäger als Vorstand gegründet. Sein Nachfolger, der geniale Ernst Fuchs, der aus der 1. Augenklink kam, hat Wien von 1885-1915 weltweit zum Zentrum der ophthalmologischen Lehre gemacht, worauf wir stolz sein können. Daneben bestand aber auch 
ein heftiger Krieg in der Ausrichtung der Spezifizierung der Augenkliniken. War die 1. Augenklinik seit Arlt pathologisch-anatomisch orientiert, so war die 2. Augenklinik von Jäger von Jaxtthal die physikalisch-optisch ausgerichtete. Welcher Verlust an wissenschaftlichem Potential!

Erst durch Dimmer, der zuerst 5 Jahre der 1. Augenklinik vorstand und dann für weitere 10 Jahre zum Leiter der 2. Augenklinik berufen wurde, kam es zu einer Harmonisierung beider Bereiche. Ohne die Kombination von Wissen aus beiden Gebieten - Pathologie und Optik - wären wohl kaum die ersten Fundusfotos verschiedener retinaler Pathologien entstanden, damals eine bahnbrechende Errungenschaft.

Meller widmete sich als Vorstand der 1. Augenklinik der Augenchirurgie, was sich auch in seinem primär in englischer Sprache veröffentlichten Handbuch „Ophthalmic Surgery“ niederschlug, die erste Monographie über Operationen am Auge überhaupt und für lange Zeit „das Lehrbuch“ im anglo-amerikanischen Raum, während Lindner als Chef der 2. Univ.-Augenklinik sich primär mehr mit der Erforschung bakterieller Augenerkrankungen beschäftigte und später ganz wesentlich zur Erforschung der Netzhaut- und Glaskörpererkrankungen beitrug. Ihm folgte Böck, an der 1. Augenklinik war Pillat Vorstand. Besonders die Sitzungen der Wiener ophthalmologischen Gesellschaft dienten als Rahmen wissenschaftlicher Streitgespräche der beiden Klinikchefs. Jene zwischen Hruby und Böck habe ich selbst erleben dürfen. Als Assistenzärzte der einen oder anderen Klinik, die damals in ihrer Karriere völlig abhängig von der Entscheidung des Klinikchefs waren, legten wir furchtsam unsere Ohren zurück.
Bis zur Zusammenlegung beider Kliniken mit der Übersiedelung in das neue AKH bestand ein mehr oder weniger heftiger Konkurrenzkampf zwischen den beiden Augenkliniken in Wien, manchmal befruchtend, meist aber hemmend für internationale Wissenschaft und Innovation.

Kürzlich gab es eine Diskussion eines internationalen Senders, ob Wissenschaft heute in zunehmendem Maße politisiert wird und ob der Einfluss der Forscher auf die Wahl ihrer Themen im Vergleich zum Einfluss politischer Entscheidungsträger und Geldgeber immer geringer werde. Alle Diskussionsteilnehmer waren sich einig, dass dies bereits der Fall sei.

Die Uneinigkeit zwischen den Forschern, Neid und Intrigen schwächen klar unser Potential. Talente bleiben brach liegen oder werden mutwillig zerstört. Wichtige Entscheidungen werden dann auf anderen Ebenen getroffen, bei denen Mediziner und Forscher nichts mehr zu sagen haben. Da wir kaum aus der Geschichte lernen, sieht so unsere Zukunft aus.

Ihre

Univ.-Prof. Dr. Susanne Binder

Editor in Chief

\section{Interessenkonflikt}

Es besteht kein Interessenkonflikt.

Open Access Dieser Artikel unterliegt den Bedingungen der Creative Commons Attribution License. Dadurch sind die Nutzung, Verteilung, und Reproduktion erlaubt, sofern der/die Originalautor/en und die Quelle angegeben sind. 